\title{
ON A QUESTION OF BOURAS CONCERNING WEAK COMPACTNESS OF ALMOST DUNFORD-PETTIS SETS
}

\author{
JIN XI CHEN AND LEI LI
}

\begin{abstract}
We give a positive answer to the question of K. Bouras ['Almost Dunford-Pettis sets in Banach lattices', Rend. Circ. Mat. Palermo (2) 62 (2013), 227-236] concerning weak compactness of almost Dunford-Pettis sets in Banach lattices. That is, every almost Dunford-Pettis set in a Banach lattice $E$ is relatively weakly compact if, and only if, $E$ is a $K B$-space.
\end{abstract}

Let $E$ be a Banach lattice. Recall that a norm bounded subset $A$ of $E$ is called to be $L$-weakly compact if $\left\|x_{n}\right\| \rightarrow 0$ for every disjoint sequence $\left(x_{n}\right)$ contained in the solid hull of $A$. Every $L$-weakly compact set is relatively weakly compact set, but the converse does not hold in general. In an $L$-space, $L$-weakly compact sets and relatively weakly compact sets coincide. More generally, every relatively weakly compact subset of $E$ is $L$-weakly compact if, and only if, $E$ has the positive Schur property. Hereby, we say a Banach lattice $E$ has the positive Schur property if every weakly null sequence with positive terms in $E$ is norm null.

Following Bouras [2], a bounded subset $A$ of the Banach lattice $E$ is said to be an almost Dunford-Pettis set if every disjoint weakly null sequence $\left(f_{n}\right)$ of $E^{\prime}$ converges uniformly to zero on $A$, that is, $\sup _{x \in A}\left|f_{n}(x)\right| \rightarrow 0$. In [2], Bouras showed that every $L$-weakly compact set in a Banach lattice $E$ is necessarily almost Dunford-Pettis. Also, every relatively weakly compact set in $E$ is almost Dunford-Pettis if and only if $E$ has the weak Dunford-Pettis property. If every almost Dunford-Pettis set in $E$ is relatively weakly compact (in particular, $L$ weakly compact), then $E$ is a $K B$-space [2, Theorem 2.10]. Conversely, if $E$ is an $L$-space, or a dual $K B$-space, or the norm of $E^{\prime \prime}$ is order continuous, then every almost Dunford-Pettis set in $E$ is $L$-weakly compact (and hence relatively weakly compact). In those three cases, $E$ is a $K B$-space. Therefore, Bouras posed the following open question.

Question [2]. Does the assumption " $E$ is a $K B$-space" imply that each almost Dunford-Pettis set in $E$ is relatively weakly compact (resp. $L$-weakly compact)?

2010 Mathematics Subject Classification. Primary 46B42; Secondary 46A40, 46B50.

$K e y$ words and phrases. almost Dunford-Pettis set, $L$-weakly compact set, $K B$-space, Banach lattice.

This work was supported by NSFC (No.11301285). The first author was also supported by China Scholarship Council (CSC) and was visiting the University of South Carolina when this work was completed. 
In this short note, we give a positive answer to the question with respect to weak compactness of almost Dunford-Pettis sets. That is, every almost DunfordPettis set in a Banach lattice $E$ is relatively weakly compact if, and only if, $E$ is a $K B$-space.

For Banach lattice theory, we refer the reader to [1, 4] and also the original paper of Bouras [2].

Theorem. Let $E$ be a Banach lattice. Every almost Dunford-Pettis set in $E$ is relatively weakly compact if, and only if, $E$ is a $K B$-space.

Proof. For the proof of the "only if" part see Theorem 2.10 of [2]. We need only to prove the "if" part. To this end, let $E$ be a $K B$-space and $A$ an almost Dunford-Pettis set in $E$.

First, we claim that the solid hull $\operatorname{sol}(A)$ of $A$ is likewise almost Dunford-Pettis. Otherwise, there would exist a disjoint weakly null sequence $\left(f_{n}\right) \subseteq E^{\prime}$ such that $\sup _{x \in \operatorname{sol}(A)}\left|f_{n}(x)\right|>\varepsilon_{0}$ for some $\varepsilon_{0}>0$ and all $n \in \mathbb{N}$. So, we can find two sequences $\left(x_{n}\right) \subseteq A$ and $\left(y_{n}\right) \subseteq \operatorname{sol}(A)$ satisfying

$$
\left|y_{n}\right| \leq\left|x_{n}\right|, \quad 0<\varepsilon_{0}<\left|f_{n}\left(y_{n}\right)\right| \leq\left|f_{n}\right|\left(\left|x_{n}\right|\right)
$$

for each $n$. Again, by [1, Theorem 1.23] there exists a sequence $\left(g_{n}\right) \subseteq E^{\prime}$ such that

$$
\left|g_{n}\right| \leq\left|f_{n}\right|, \quad\left|g_{n}\left(x_{n}\right)\right|>\varepsilon_{0} .
$$

Clearly, $\left(g_{n}\right)$ is a disjoint sequence. From the weak convergence of $\left(f_{n}\right)$ it follows that $g_{n} \stackrel{w}{\rightarrow} 0$ (cf. [1, Thoerem 4.34]). Since $A$ is almost Dunford-Pettis, we have

$$
\varepsilon_{0}<\left|g_{n}\left(x_{n}\right)\right| \leq \sup _{x \in A}\left|g_{n}(x)\right| \rightarrow 0
$$

which is impossible. This proves that $\operatorname{sol}(A)$ is an almost Dunford-Pettis set.

Now we can assume without loss of generality that $A$ is a solid almost DunfordPettis set in $E$. Let $\rho_{A}(f):=\sup _{x \in A}\langle|f|,|x|\rangle$ for each $f \in E^{\prime}$. Clearly, $\rho_{A}$ is a lattice seminorm on $E^{\prime}$, and $\rho_{A}(f)=\sup _{x \in A}|f(x)|$. Let $\left(f_{n}\right)$ be an arbitrary order bounded disjoint sequence in $E^{\prime}$. Since $f_{n} \stackrel{w}{\rightarrow} 0$ and $A$ is almost DunfordPettis, we have $\rho_{A}\left(f_{n}\right) \rightarrow 0$. Therefore, for every $0 \leq f \in E^{\prime}$, by [4, Theorem 2.3.3] $A$ is approximated order bounded with respect to $\rho_{f}$, that is to say, for every $\varepsilon>0$ there exists $0 \leq x \in E$ satisfying

$$
A \subseteq[-x, x]+\varepsilon B_{\rho_{f}}
$$

where $B_{\rho_{f}}=\left\{x \in E: \rho_{f}(x)=f(|x|) \leq 1\right\}$.

Note that the $w^{*}$-closure $\bar{A}^{w^{*}}$ of $A$ in $E^{\prime \prime}$ is $w^{*}$-compact. To prove that $A$ is relatively weakly compact in $E$ it suffices to show that $\bar{A}^{w^{*}} \subseteq E$. Since $E$ is a $K B$-space, we have $E=\left(E^{\prime}\right)_{n}^{\prime}$ (cf. [1, Theorem 4.60]). Thus, we have to show that every element $x^{\prime \prime}$ of $\bar{A}^{w^{*}}$ is an order continuous linear functional on $E^{\prime}$, 
equivalently, $\left|x^{\prime \prime}\right| \in\left(E^{\prime}\right)_{n}^{\prime}$. To this end, let $\left(f_{\alpha}\right)$ be an arbitrary decreasing net in $\left(E^{\prime}\right)^{+}$with $f_{\alpha} \downarrow 0$. It is enough to show that $\left\langle\left|x^{\prime \prime}\right|, f_{\alpha}\right\rangle \downarrow 0$. For each $\alpha$ we have

$$
\begin{aligned}
\left\langle\left|x^{\prime \prime}\right|, f_{\alpha}\right\rangle=\sup \left\{\left|\left\langle x^{\prime \prime}, g\right\rangle\right|:|g| \leq f_{\alpha}\right\} & \leq \sup \left\{|\langle g, x\rangle|:|g| \leq f_{\alpha}, x \in A\right\} \\
& \leq \rho_{A}\left(f_{\alpha}\right)
\end{aligned}
$$

To finish the proof, we need only to prove that $\rho_{A}\left(f_{\alpha}\right) \downarrow 0$. Indeed, it may be assumed that there exists an element $f \in E^{\prime}$ such that $0 \leq f_{\alpha} \leq f$ for all $\alpha$. Let $\varepsilon>0$ be fixed. By $(*)$ we have $\rho_{A}\left(f_{\alpha}\right) \leq f_{\alpha}(x)+\varepsilon$ for all $\alpha$. Note that $f_{\alpha}(x) \downarrow 0$. It follows that $\inf _{\alpha} \rho_{A}\left(f_{\alpha}\right) \leq \varepsilon$. Since $\varepsilon>0$ is abitrary, we have $\inf _{\alpha} \rho_{A}\left(f_{\alpha}\right)=0$, as desired.

In a $K B$-space without the weak Dunford-Pettis property (e.g., $\ell_{p}, 1<p<\infty$ ) almost Dunford-Pettis sets and relatively weakly compact sets can not coincide.

There is another topic included in Bouras' question:

(Q1) Is every almost Dunford-Pettis set in a KB-space L-weakly compact?

We are not able to answer it and have to leave it still open. However, we can make some comments on (Q1). In [3] Chen et al. introduced the class of almost limited sets in Banach lattices. A norm bounded subset $A$ of a Banach lattice $E$ is said to be an almost limited set if every disjoint, weak* null sequence $\left(f_{n}\right)$ of $E^{\prime}$ converges uniformly to zero on $A$. Clearly, every almost limited set in $E$ is an almost Dunford-Pettis set. Almost limited sets and $L$-weakly compact sets coincide in $E$ if and only if the norm of $E$ is order continuous ([3, Theorem $2.6(2)])$. Therefore, (Q1) is the same as the following question:

(Q1') Do almost Dunford-Pettis sets and almost limited sets coincide in a KBspace?

Let us recall that a Banach lattice $E$ has the positive Schur property if, and only if, every relatively weakly compact subset of $E$ is $L$-weakly compact. We know that every Banach lattice with the positive Schur property is a $K B$-space with the weak Dunford-Pettis property. In 1994 Wnuk [5] left a question which is still open until now:

(Q2) Does every KB-space with the weak Dunford-Pettis property have the positive Schur property?

By the Theorem of the present paper, in a $K B$-space with the weak DunfordPettis property almost Dunford-Pettis sets and relatively weakly compact sets coincide. Hence, if the answer to (Q1) is positive, then the answer to (Q2) is likewise positive. In other words, if the answer to (Q2) were negative, then there would exist a $K B$-space where we can find an almost Dunford-Pettis set which is not $L$-weakly compact. 


\section{REFERENCES}

[1] C. D. Aliprantis and O. Burkinshaw, Positive Operators (reprint of the 1985 original), Springer, Dordrecht, 2006.

[2] K. Bouras, Almost Dunford-Pettis sets in Banach lattices, Rend. Circ. Mat. Palermo (2) 62 (2013), 227-236.

[3] J. X. Chen, Z. L. Chen and G. X. Ji, Almost limited sets in Banach lattices, J. Math. Anal. Appl. 412 (2014), 547-553.

[4] P. Meyer-Nieberg, Banach Lattices, Universitext, Springer-Verlag, Berlin, 1991.

[5] W. Wnuk, Banach lattices with the weak Dunford-Pettis property, Atti Semin. Mat. Fis. Univ. Modena 42 (1994), 227-236.

Department of Mathematics, Southwest Jiaotong University, Chengdu 610031, PR CHINA

E-mail address: jinxichen@home.swjtu.edu.cn

Department of Mathematics, Nankai University, Tianjin 300071, PR China

E-mail address: leilee@nankai.edu.cn 